\title{
Is the List of Incomplete Open Cubes Complete?
}

\author{
Natasha Rozhkovskaya ${ }^{1} \cdot$ Michael Reb $^{1}$
}

Published online: 16 July 2015

(C) Kim Williams Books, Turin 2015

\begin{abstract}
Variations of Incomplete Open Cubes is the major project by the twentieth-century conceptual artist Sol LeWitt. In this paper we interpret the enumerative component of the project as embeddings of graphs. This formulation permits use of an algorithm to check the completeness of the list of the structures produced by the artist. Our conclusion is that the artist found the correct number of structures (that is, 122), but that his list contains a mistake in the presentation of a pair of incomplete cubes, a discovery that appears not to have been noted before.
\end{abstract}

Keywords Graph theory · Open cubes · Sol LeWitt · Combinatorics

\section{Introduction}

For many decades wall drawings and structures of the legendary American artist Solomon LeWitt (1928-2007) have inspired artists, astonished art lovers, and challenged scholars to analyze the artist's concepts and ideas. LeWitt worked with various types of media, including photography, drawing, and painting. Even though he is first and foremost a conceptual artist, his art has evident connections with architecture. Some scholars note the similarity of the artist's philosophy with the creative process of an architect. Moreover, early in his career LeWitt worked as a designer for I. M. Pei's office; much later the artist collaborated with the architect Stephen Lloyd on a design of the reform synagogue for the Congregation Beth Shalom Rodfe Zedek in Chester, Connecticut.

Natasha Rozhkovskaya

rozhkovs@math.ksu.edu

Michael Reb

mreb@ksde.org

1 Department of Mathematics, Kansas State University, Manhattan, KS 66506, USA 
Many of the mathematical components of LeWitt's designs appeal to combinatorial questions that can be formulated as "lists all possible cases". In some projects the total number of possibilities can be computed without any effort by well-known formulas, such as numbers of combinations, numbers of permutations, powers of numbers, etc. In what follows we will review briefly two examples. In other cases enumeration complexity may go beyond the standard formulas of a basic college textbook on discrete mathematics. As an example of such complexity, we consider Variations of Incomplete Open Cubes.

\section{Combinatorial Description of LeWitt's Projects}

\section{Wall \#260: All Combinations of Arcs from Corners and Sides; Straight, Not-Straight, and Broken Lines, 1973}

A photograph of Wall \#260 can be found on (LeWitt 1975) (see also Legg 1978; LeWitt 1994, 2009). This design was used several times for wall drawing installations at different exhibitions. The wall is divided by a square grid, and each square contains a pair of lines. Five types of lines are used to create the series: the concave up and down arcs of a circle, a straight line, a dashed line, and a freehandstyle line. Each of these lines can connect the opposite corners or the opposite midpoints of the sides of a square of the grid. The wall drawing of this project consists of all pairs of two lines of different types. Figure 1 summarizes five types of lines, four directions and provides a detail of a design with an example of nine squares of the grid. The combinatorics of this project is clear: five types of lines in four directions give us twenty basic elements, and the number of unordered pairs of these twenty basic elements is described by $\left(\begin{array}{c}20 \\ 2\end{array}\right)=190$ combinations. The whole design, indeed, consists of 190 squares.
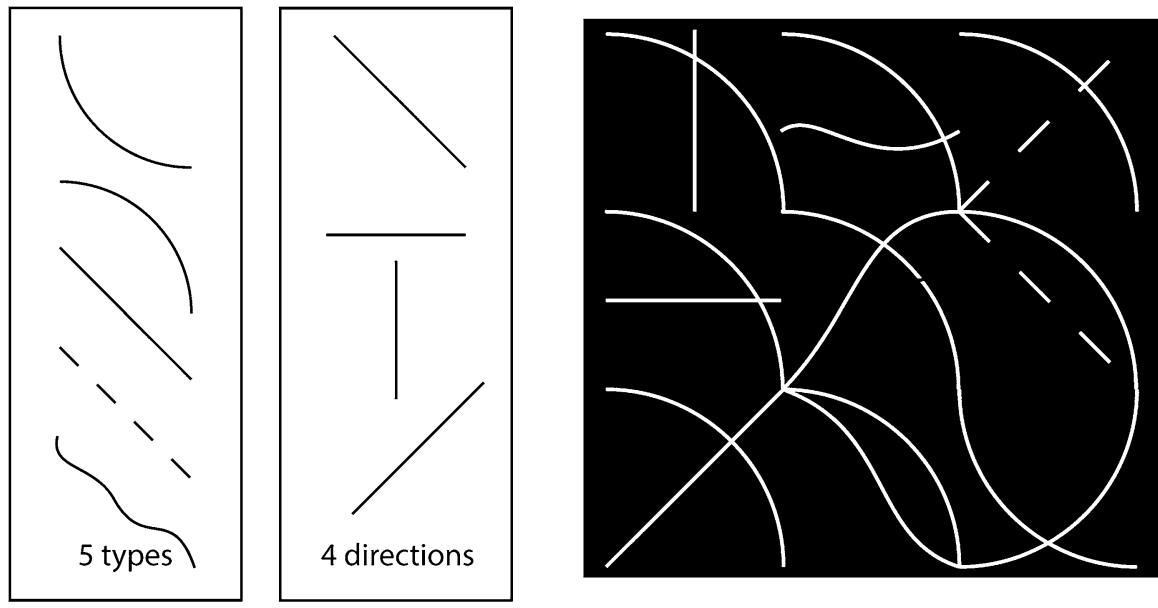

Fig. 1 Wall \#260: fundamental elements of the pattern and a detail of a design with an example of nine combinations 

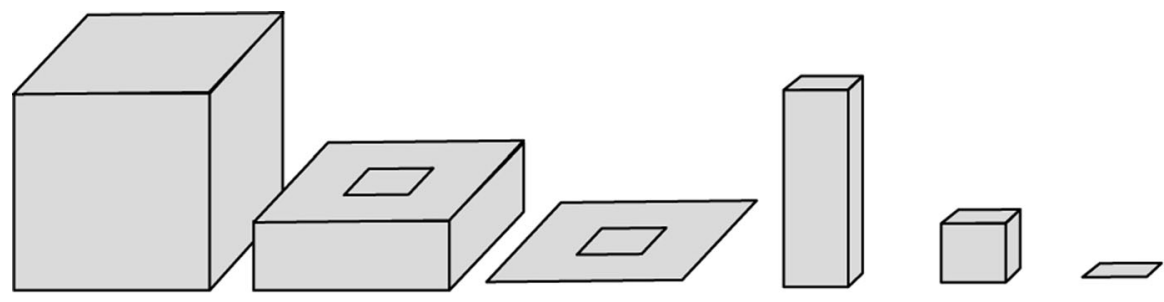

Fig. 2 Closed outside and closed inside structures

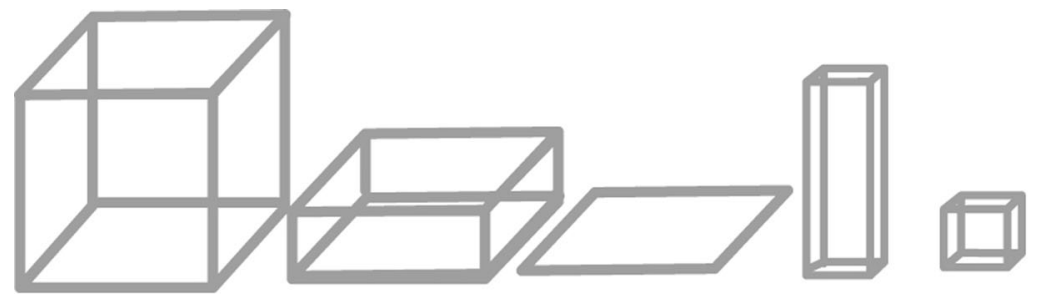

Fig. 3 Open outside and open inside structures

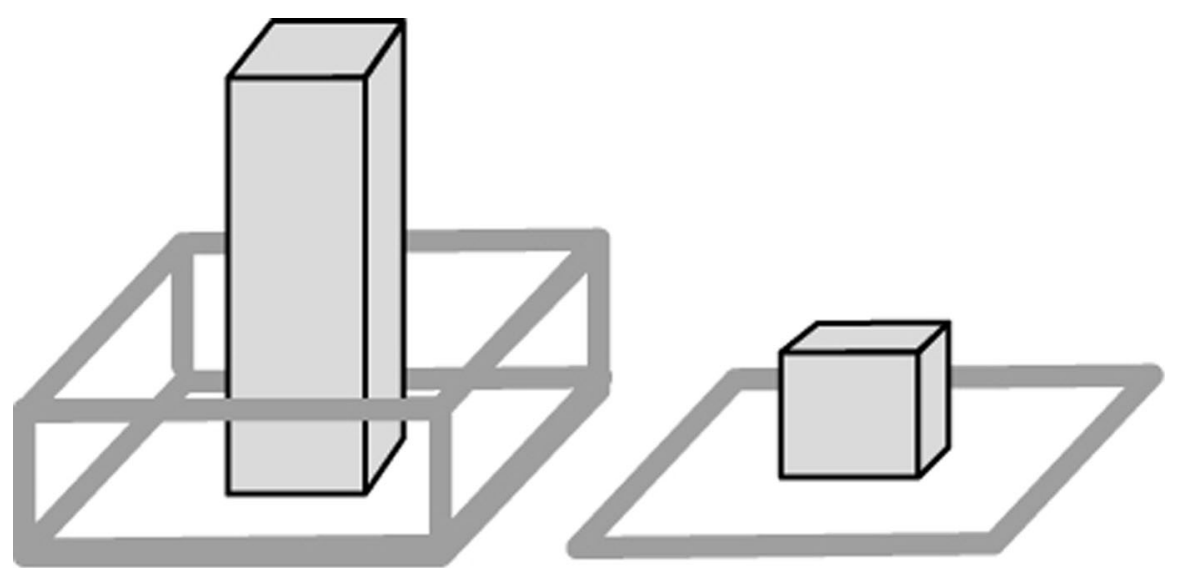

Fig. 4 Two out of thirty-six possible nested pairs of structures

\section{Serial Project No. 1 (ABCD), 1966}

A photograph of this project can be found on the MoMA website (LeWitt 1966). This installation consists of all pairs of nested parallelepipeds that satisfy the following properties. In each pair the outside parallelepiped has the square base of dimension $3 \times 3$ units, the inside parallelepiped has the square base of dimension $1 \times 1$ units. The heights of parallelepipeds can vary as 0,1 or 3 units, and each parallelepiped can be either "open" (consisting of edges only) or "closed" (all faces are present) (Figs. 2, 3). Thus, in each pair, the outside parallelepiped and the inside parallelepiped each have six options (three height values and closed/open) and the total number of structures is $6^{2}=36$ (Fig. 4). 
The title $A B C D$ stands for the letter notation of four types of groups by the open/closed characteristic: A (outside open, inside open), B (outside open, inside closed), C (outside closed, inside open), and D (outside closed, inside closed).

\section{Incomplete Open Cubes, 1974}

Variations of Incomplete Open Cubes is recognized as one of the artist's major projects. It is represented by an installation of 122 frame structures supplemented by drawings and diagrams of the structures. The catalogue (LeWitt 1974) summarizes the photos and the diagrams of the project. The rules followed by LeWitt for production of Incomplete Open Cubes are quite simple: each individual structure of the project is a cube with some edges removed, with the following rules observed:

- the structure should be three-dimensional. For example, a square, or a single edge are not listed in the project, since they are not three-dimensional;

- the structure should be connected;

- two structures are considered to be identical if one can be transformed into another by a space rotation (but not by a reflection).

For example, the two structures in Fig. 5 represent two different incomplete open cubes, since one of them is a reflection of the other:

However, all the structures in Fig. 6 represent the same incomplete open cube, since they differ from each other only by rotations:

Note that the choice of restrictions on the structures is very natural. The artist aimed to create a unified group of objects, and the introduction of two-dimensional elements into the set of three-dimensional structures would break the unified look of the collection. Therefore, one- and two-dimensional incomplete cubes are not included in the project. Note further that production and installation of disconnected structures would require sophisticated technical solutions, so it is natural to remove from the list disconnected incomplete open cubes. Finally, some rotations place

Fig. 5 Two different incomplete cubes

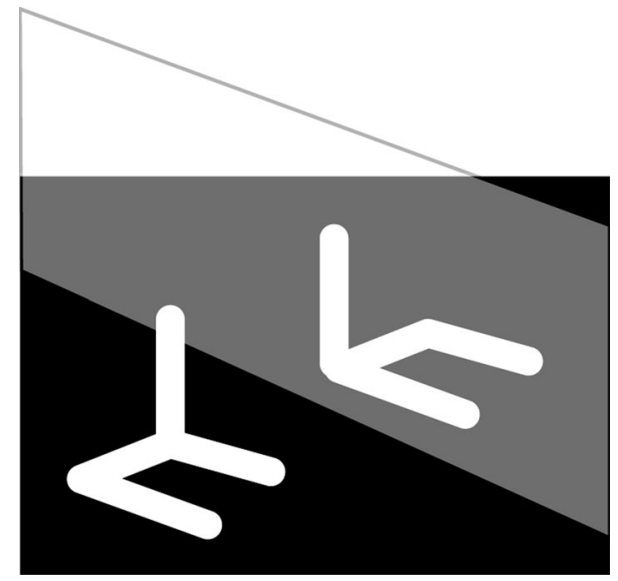




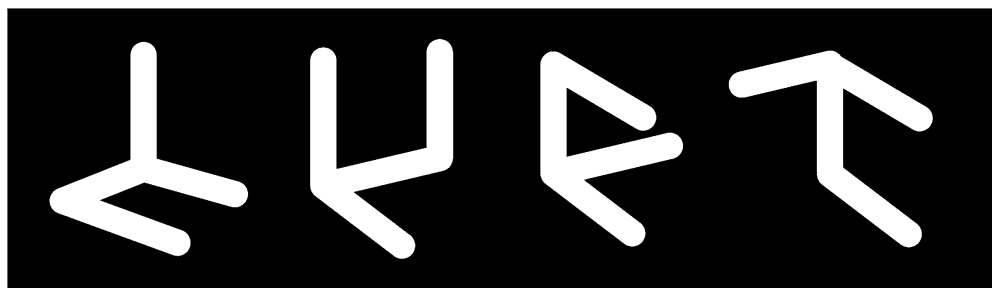

Fig. 6 The same incomplete open cube rotated

some structures in unstable positions, and the last restriction on the structures helps to resolve this difficulty as well.

The whole project consists of 122 structures, and even at the first glance, it is clear that combinatorial description of this project is far from trivial.

According to (LeWitt 2001), Sol LeWitt did not realize the full complexity of the corresponding mathematical problem at the beginning of the project, but once he started, he wanted to solve it completely. His response to the question of why he didn't ask a professional mathematician for help, was, "In the first place, I thought it'd be so easy that it wouldn't be necessary. Secondly, I didn't know any mathematician to ask. Thirdly, it was a kind of challenge to be able to do it and to work it all out. It got to be a game or a puzzle that I wanted to solve" (LeWitt 2001, p. 25.)

He organized the list of incomplete cubes in groups according to the number of edges of the structures. In order to check that there were no repetitions, the artist made small three-dimensional models. The final version of the list was used to produce a series of large aluminum sculptures that was accompanied by drawings of diagrams in different presentations.

Two questions arise naturally with regard to this work:

- How could the list produced by the artist be checked for possible mistakes?

- Is the list complete?

Here we use the methods of graph theory to answer these questions. These methods allow us to formulate the art project precisely in the language of mathematics, to notice a minor mistake, and to compare Incomplete Open Cubes with some developments in modern combinatorics.

\section{Graph Theory}

Let us briefly outline some notions and definitions of the graph theory. ${ }^{1}$ A graph $G$ is an ordered pair $(V, E)$ of two sets. The first set $V$ is a finite set of elements called vertices $V=V(G)=\{u, v, \ldots\}$; the second set $E=E(G)$ is the set of edges, that is, a set of unordered pairs of distinct vertices. It is common to represent vertices of graphs by dots on a plane, and edges by segments of curves that connect them (Fig. 7).

\footnotetext{
${ }^{1}$ For more details we refer the reader to (Brualdi 2010).
} 

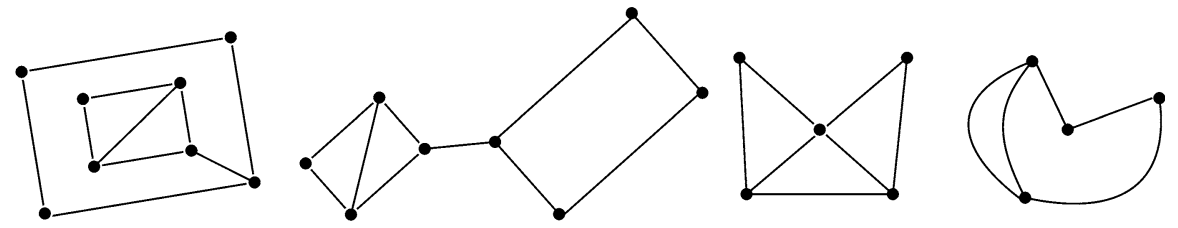

Fig. 7 Examples of graphs
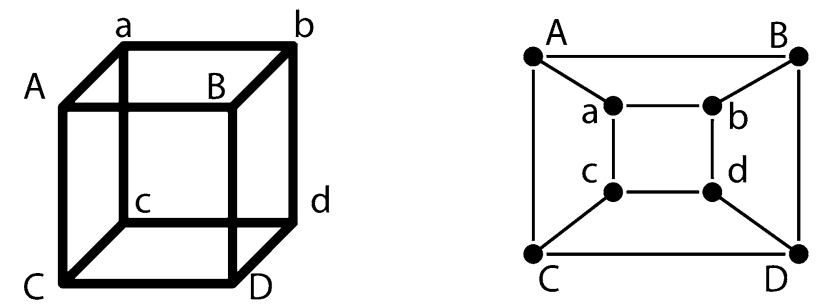

Fig. 8 Planar graph of $I^{3}$

The degree of a vertex is the number of edges adjacent to that vertex. A graph is called bipartite if the set of all vertices of the graph can be divided into two types, so that no edge connects two vertices of the same type. Recall that a unit cube is the set $\left\{\left(x_{1}, \ldots, x_{n}\right) \in \mathbb{R}^{n}: 0 \leq x_{i} \leq 1, i=1, \ldots, n\right\}$. Vertices of the unit cube are the points with coordinates $\left(x_{1}, \ldots, x_{n}\right)$, where $x_{i}$ are only $0 \mathrm{~s}$ or $1 \mathrm{~s}$. The vertices and the edges of the unit cube define naturally a graph, which we denote as $I^{n}$. For example, threedimensional cube $I^{3}$ can be realized as a planar bipartite graph (Fig. 8).

One of the common questions studied by graph theory regards the embedding of certain classes of graphs into graphs of another type. An embedding of a graph $G$ in a graph $G^{t}$ is an edge-preserving, one-to-one mapping from the set of vertices $V(G)$ to the set of vertices $V\left(G^{t}\right)$. The image of $G$ under an embedding is an isomorphic to $G$ subgraph of $G^{t}$. Here are examples of some embeddings of a given graph in $I^{3}$ (Fig. 9).

Graphs that can be embedded into an $n$-dimensional cube $I^{n}$ are traditionally called cubical graphs. The minimal dimension $n$ such that $I^{n}$ admits such embedding, is called the dimension. Our main interest is cubical graphs with a maximum dimension of three. Recall that the group of symmetries of $I^{3}$ has the order 48 and is generated by rotations and reflections of the cube. The subgroup generated by rotations only has the order 24 and is isomorphic to the group of
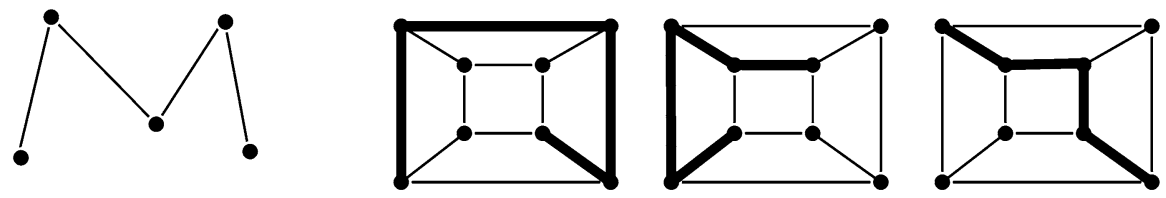

Fig. 9 Several embeddings of the same graph in $I^{3}$ 
permutations of four elements. It acts by permutations on the set of four diagonals of the cube. Now we are ready to formulate the Incomplete Open Cubes project in the language of graph theory.

Incomplete Open Cubes Problem. Classify all Three-Dimensional Embeddings of Cubical Graphs in $I^{3}$, Up to Rotations of $I^{3}$

This formulation immediately implies that in order to obtain the classification we have to perform the following two steps:

1. First, we have to understand, what graphs can be embedded in $I^{3}$. For example, Fig. 10 shows examples of graphs that can be embedded in $I^{3}$, and Fig. 11 shows examples that cannot be embedded in $I^{3}$.

2. Second, for each isomorphism class of cubical graphs we list separately its embeddings in $I^{3}$ (up to rotations of the cube): we have already seen above that the same graph can be embedded in $I^{3}$ in several ways. Thus, for each graph that can be embedded in $I^{3}$, we must list all possible embeddings.

The answer to the first step is the following: there are 63 graphs that can be embedded in $I^{3}$. We would like to remark that, as with many other problems in graph theory, it is very unlikely that there is a closed formula that would produce this answer. Rather, a simple algorithm can be used here: starting from the full cube $I^{3}$, at each step remove one of the remaining edges and compare the isomorphism class of the resulting graph with the ones already obtained by removing some other edges. The outcome of such algorithm is summarized in Fig. 12: two isomorphism classes are connected in the diagram if and only if they differ from each other by exactly one edge.

An alternative way to obtain these 63 graphs is to use one of existing databases of graphs, such as (Read and Wilson 1998). Note that any graph embeddable in $I^{3}$ is a bipartite graph with a maximum of eight vertices and a maximum vertex degree of three. All such graphs can be found in (Read and Wilson 1998) in the table of bipartite graphs with at most eight vertices. One can easily check, which ones from the table are embeddable in $I^{3}$ to obtain the list of Fig. 12. The numerations $B 1, B 2$, etc., correspond to the names of graphs in (Read and Wilson 1998).

Observe that the graphs in Fig. 13 are not present in the Sol LeWitt's list of incomplete open cubes, since the first three cannot be embedded in $I^{3}$ as threedimensional structures, and the last one is the complete cube.

Now, for each isomorphism class in Fig. 12 we find all its embeddings (up to rotations) in $I^{3}$. Each graph has no more than six different embeddings. It is not difficult to find all of them in all cases, and the result is summarized in Fig. 14.

Recall that LeWitt organized his list in groups by the numbers of edges. In that form it is difficult to check the completeness of the list. The refinement obtained 

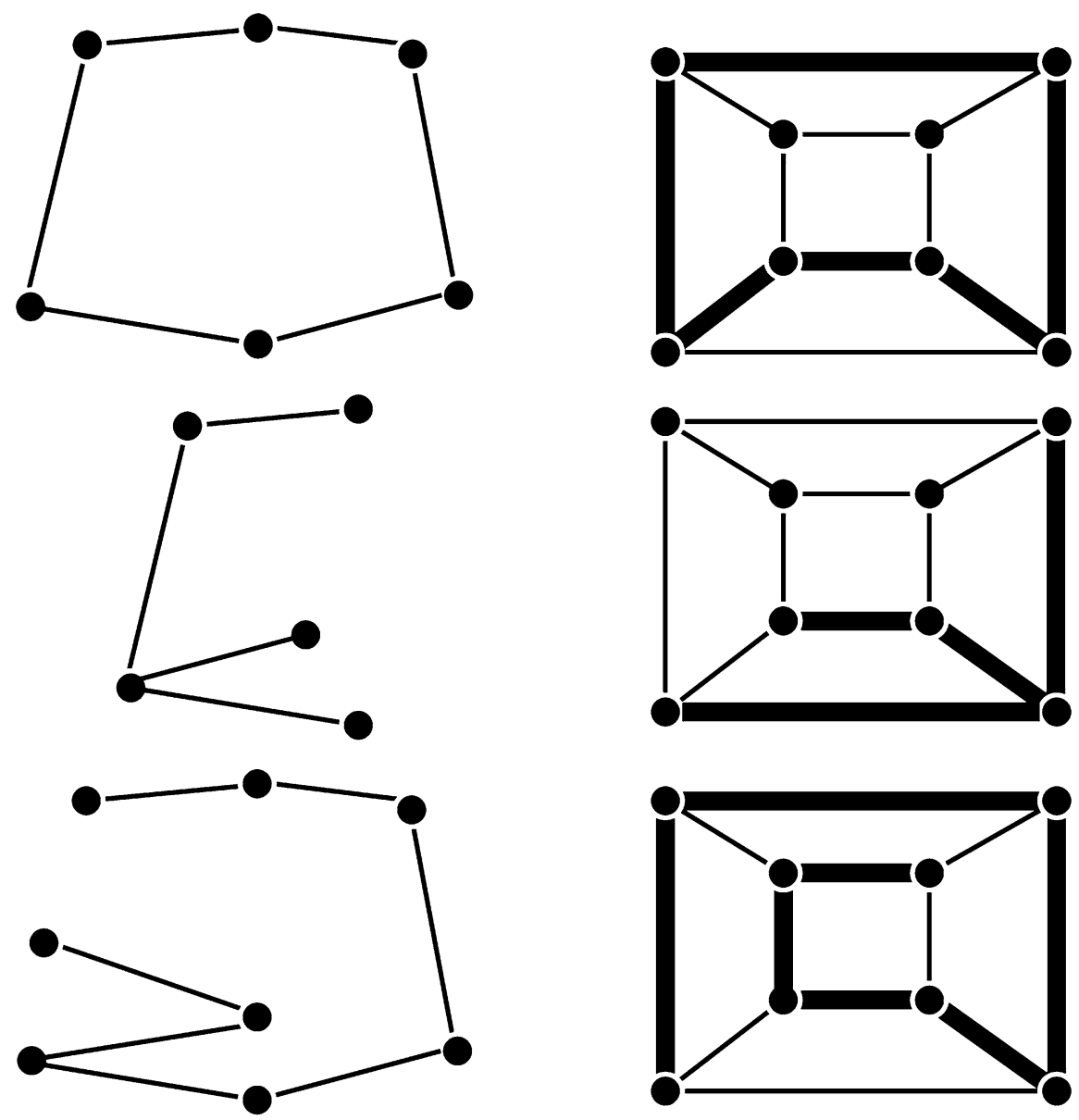

Fig. 10 Examples of graphs that can be embedded in $I^{3}$
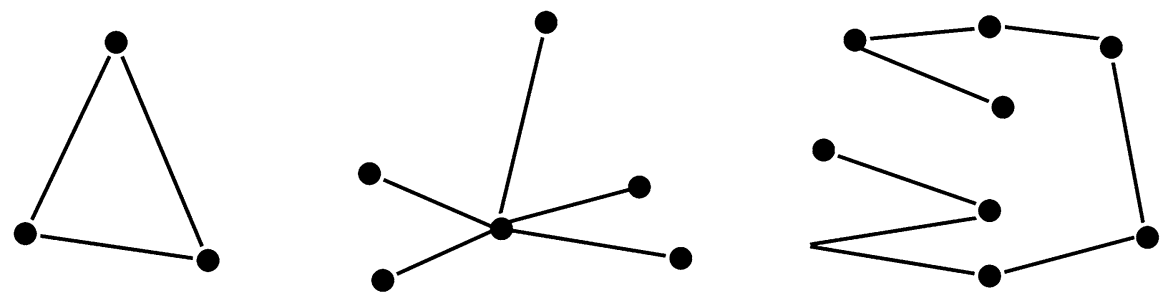

Fig. 11 Examples of graphs that cannot be embedded in $I^{3}$ 


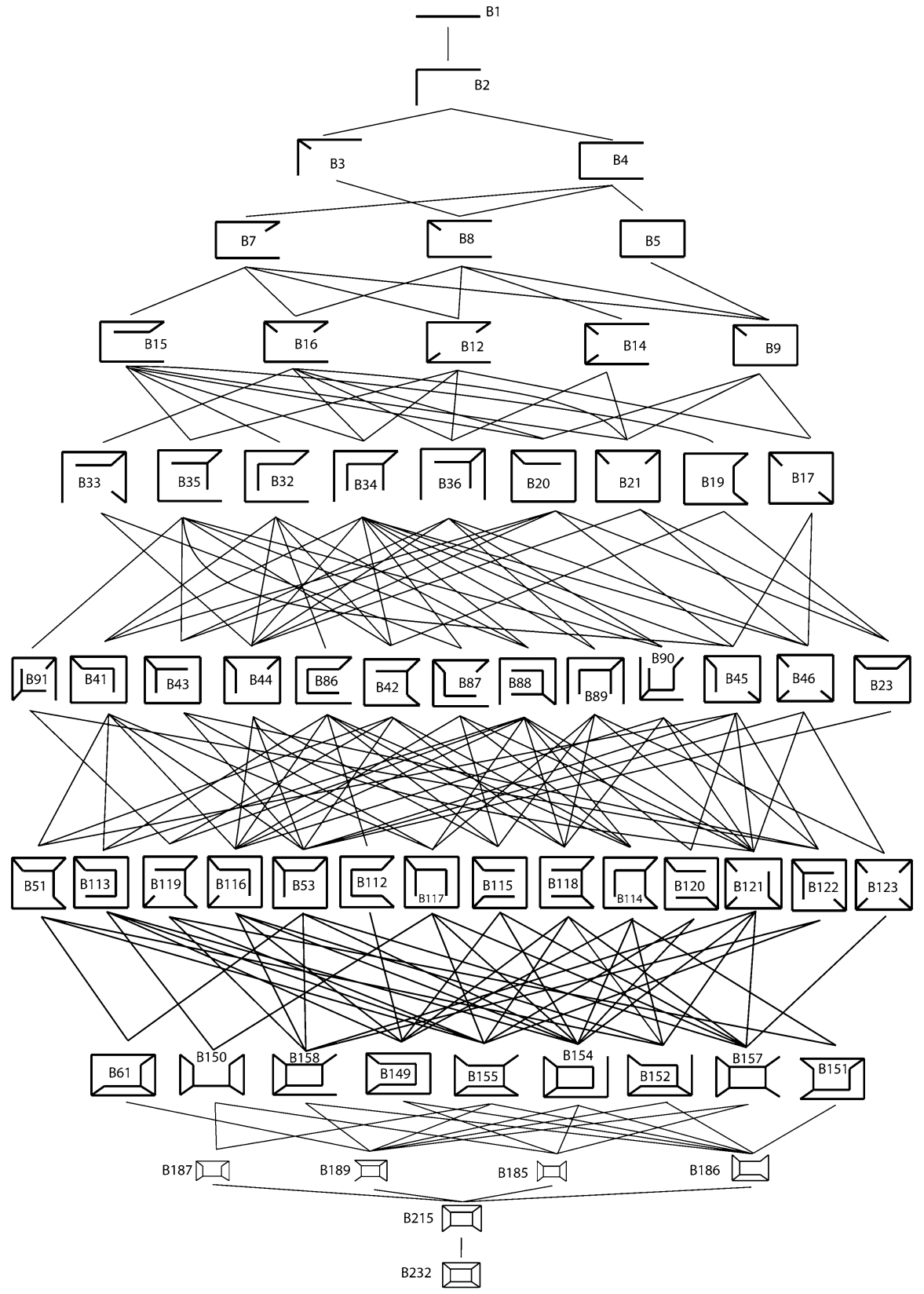

Fig. 12 Isomorphism classes of cubical graphs of a maximum dimension of three 

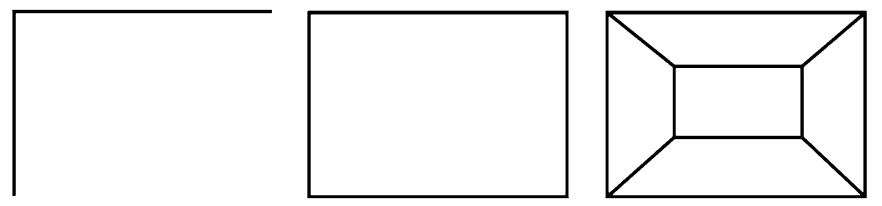

Fig. 13 Graphs of dimensions 1 or 2 and the complete cube

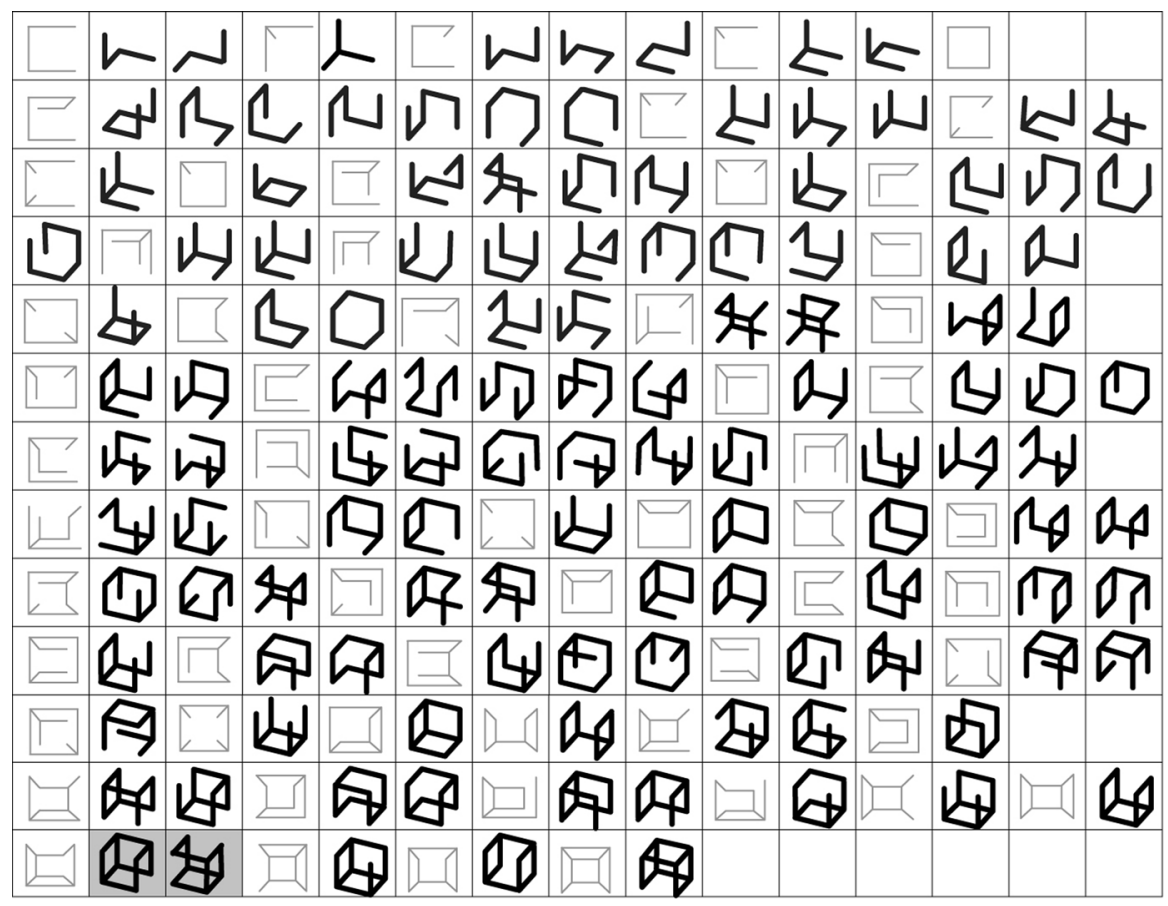

Fig. 14 Embeddings of cubical graphs in $I^{3}$

here of a classification into subgroups of isomorphism classes of corresponding graphs makes the verification much easier. This approach allowed us to confirm that there are indeed 122 different incomplete open cubes-LeWitt found all incomplete open cubes.

However, it also allowed us to notice that there is a mistake in the presentation. Namely, the artist mistakenly put the same incomplete open cube twice (the second time rotated), instead of two structures that are mirror images of each other. These are two embeddings in the shaded box in Fig. 14, which correspond to variations 

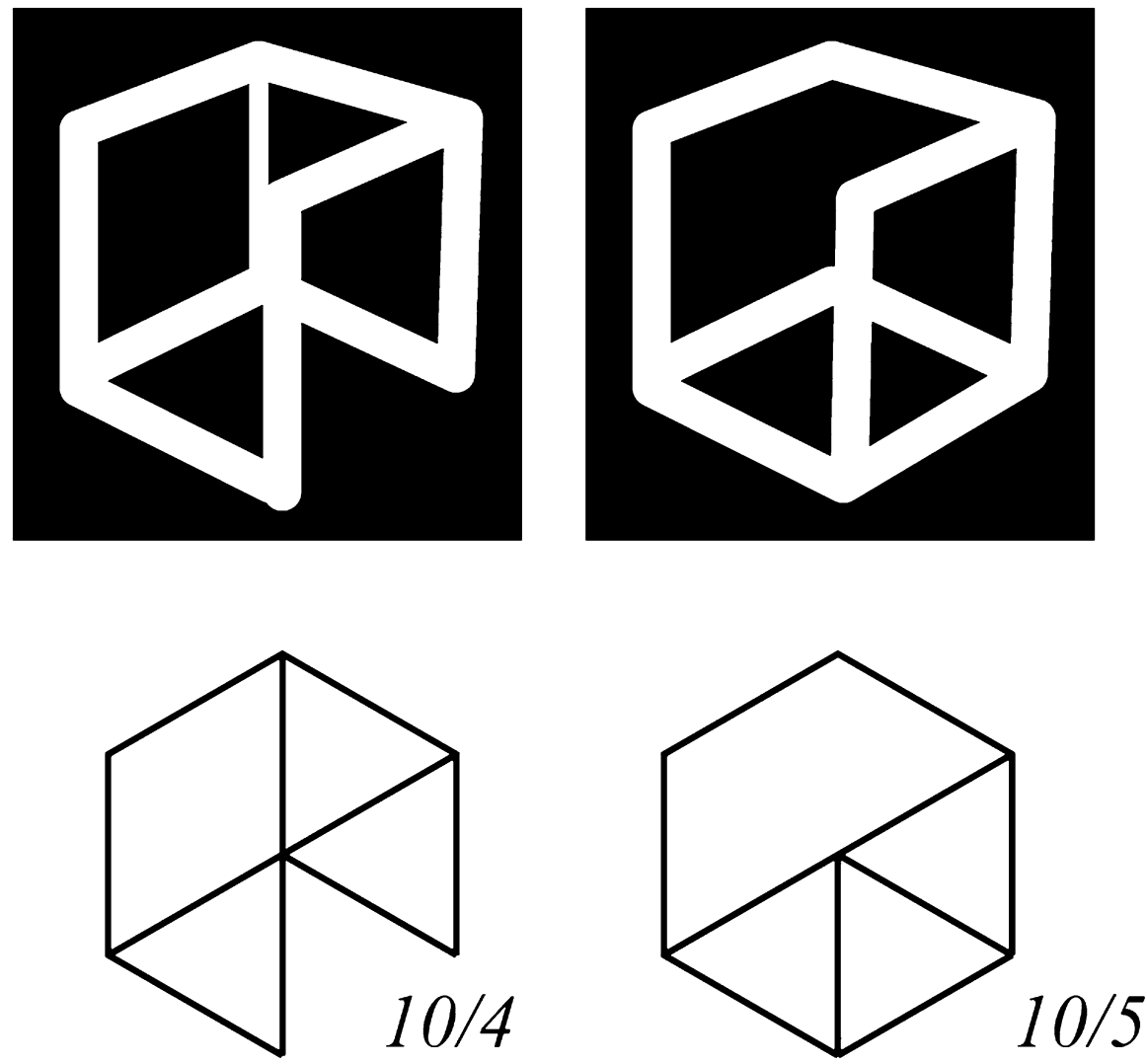

Fig. 15 Incomplete cubes $10 / 4$ and $10 / 5$ in the original Sol LeWitt's list

10/4 and 10/5 in Sol LeWitt's list. Figure 15 shows the diagrams of these two embeddings as they were presented by the artist:

Figure 16 shows the correct version, where one of the two structures in the original list is substituted by its mirror image:

\section{Conclusions}

Apparently LeWitt eventually asked professional mathematicians Erna Herrey and Arthur Babakhanian to check his list (LeWitt 2001, p. 25), and they confirmed that the number of structures is $122 .^{2}$ However, it seems that no notes on the methods used by these mathematicians were ever reproduced in any publication. Here we

\footnotetext{
2 An interesting fact is that few years earlier, in 1966, pages with some other calculations by Professor Babakhanian were presented as a part of exhibition "Working Drawings and Other Visible Things on Paper Not Necessarily to Be Viewed as Art", which was organized by another conceptual artist, Mel Bochner (Ratcliff 2000, p. 83).
} 

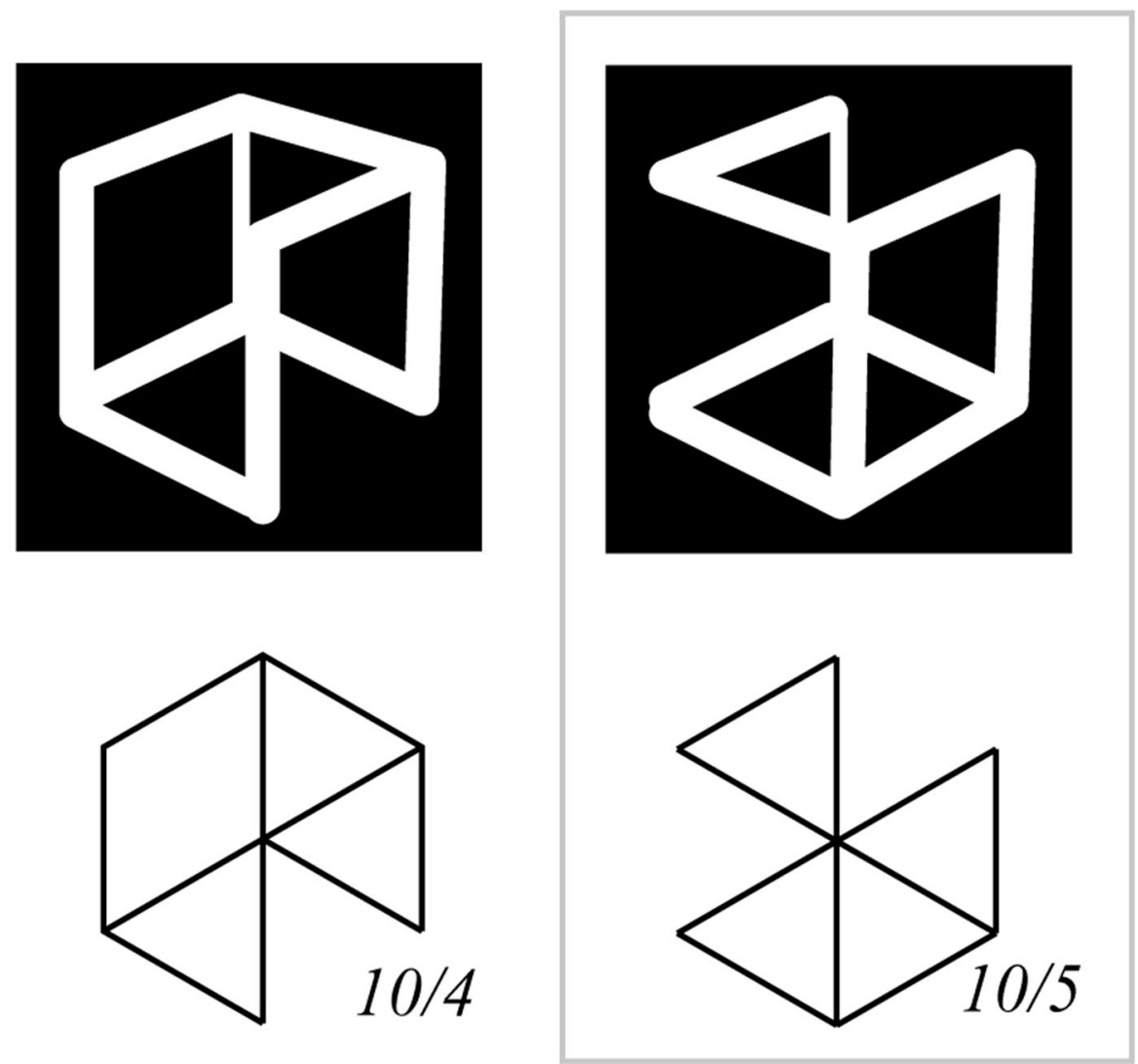

Fig. 16 Corrected versions of $10 / 4$ and 10/5

described the mathematical component of LeWitt's project and explained the ways to check the completeness of the list. As a result, the number of structures (122) is confirmed, and a minor mistake in presentation is found (to our knowledge, this has never before been observed).

\section{References}

Brualdi, Richard A. 2010. Introductory Combinatorics, 5th ed. New York: Pearson.

Legg, Alicia, ed. 1978. Sol LeWitt. New York: Museum of Modern Art.

LeWitt, Sol. 1966. Serial Project, I (ABCD). New York, Museum of Modern Art. https://www.moma.org/ learn/moma_learning/sol-lewitt-serial-project-i-abcd-1966

LeWitt, Sol. 1974. Incomplete Open Cubes. Exhibition catalogue. New York: The John Weber Gallery. LeWitt, Sol. 1975. Wall Drawing \#260, On Black Walls, All Two-Part Combinations of White Arcs from Corners and Sides, and White Straight, Not-Straight, and Broken Lines. New York, Museum of Modern Art. http://www.moma.org/collection/object.php?object_id=79898.

LeWitt, Sol. 2001. Incomplete Open Cubes. Exhibition catalogue. Hartford, Ct: Wadsworth Atheneum Museum of Art. 
LeWitt, Sol. 2009. Behind the Scenes: Installation of Focus: Sol LeWitt. Video, on view at MoMA, December 5, 2008-June 29, 2009. http://www.moma.org/explore/multimedia/videos/25/videos-all. Accessed 8 May 2015

LeWitt, Sol, Jock Reynolds, Andrea Miller-Keller. 1994. Sol LeWitt: Twenty-Five Years of Wall Drawings, 1968-1993. Seattle: University of Washington Press.

Ratcliff, Carter. 2000. Out of the Box: The Reinvention of Art, 1965-1975. New York: Allworth Press/ School of Visual Arts.

Read, Ronald C. and Robin J. Wilson. 1998. An Atlas of Graphs. Oxford: Oxford University Press.

Natasha Rozhkovskaya is an Associate Professor at Kansas State University. She received her M.S. in mathematics at Moscow State University in 1997 and her Ph.D. in mathematics at the University of Pennsylvania in 2002. Her research interests are representation theory, quantum groups, and combinatorics.

Michael A. Reb received his M.S. in mathematics at Kansas State University in 2013. He works as a senior software consultant in Information Technology at the Kansas State Department of Education. 